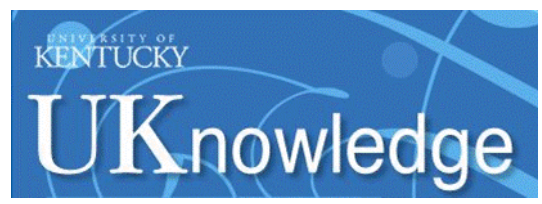

University of Kentucky

UKnowledge

\title{
Modified Single Prolonged Stress Reduces Cocaine Self- Administration During Acquisition Regardless of Rearing Environment
}

\author{
Rebecca S. Hofford \\ University of Kentucky, rebecca.hofford@uky.edu \\ Mark A. Prendergast \\ University of Kentucky, mark.prendergast@uky.edu \\ Michael T. Bardo \\ University of Kentucky, mbardo@uky.edu
}

Follow this and additional works at: https://uknowledge.uky.edu/psychology_facpub

Part of the Psychology Commons, and the Substance Abuse and Addiction Commons

Right click to open a feedback form in a new tab to let us know how this document benefits you.

\section{Repository Citation}

Hofford, Rebecca S.; Prendergast, Mark A.; and Bardo, Michael T., "Modified Single Prolonged Stress Reduces Cocaine Self-Administration During Acquisition Regardless of Rearing Environment" (2018). Psychology Faculty Publications. 165.

https://uknowledge.uky.edu/psychology_facpub/165

This Article is brought to you for free and open access by the Psychology at UKnowledge. It has been accepted for inclusion in Psychology Faculty Publications by an authorized administrator of UKnowledge. For more information, please contact UKnowledge@lsv.uky.edu. 


\section{Modified Single Prolonged Stress Reduces Cocaine Self-Administration During Acquisition Regardless of Rearing Environment}

Digital Object Identifier (DOI)

https://doi.org/10.1016/j.bbr.2017.10.023

Notes/Citation Information

Published in Behavioural Brain Research, v. 338, p. 143-152.

(C) 2017 Elsevier B.V. All rights reserved.

This manuscript version is made available under the CC-BY-NC-ND 4.0 license

https://creativecommons.org/licenses/by-nc-nd/4.0/.

The document available for download is the author's post-peer-review final draft of the article. 


\title{
Modified single prolonged stress reduces cocaine self- administration during acquisition regardless of rearing environment
}

\author{
Rebecca S. Hofford ${ }^{1}{ }^{,}$, Mark A. Prendergast $^{1}$, and Michael T. Bardo ${ }^{1}$ \\ ${ }^{1}$ Department of Psychology, University of Kentucky, Lexington KY, 40536, USA
}

\begin{abstract}
Until recently, there were few rodent models available to study the interaction of post-traumatic stress disorder (PTSD) and drug taking. Like PTSD, single prolonged stress (SPS) produces hypothalamic-pituitary-adrenal (HPA) axis dysfunction and alters psychostimulant selfadministration. Other stressors, such as isolation stress, also alter psychostimulant selfadministration. However, it is currently unknown if isolation housing combined with SPS can alter the acquisition or maintenance of cocaine self-administration. The current study applied modified SPS (modSPS; two hours restraint immediately followed by cold swim stress) to rats raised in an isolation condition (Iso), enrichment condition (Enr), or standard condition (Std) to measure changes in cocaine self-administration and HPA markers. Regardless of rearing condition, rats exposed to modSPS had greater corticosterone (CORT) release and reduced cocaine selfadministration during initial acquisition compared to non-stressed controls. In addition, during initial acquisition, rats that received both Iso rearing and modSPS showed a more rapid increase in cocaine self-administration across sessions compared to Enr and Std rats exposed to modSPS. Following initial acquisition, a dose response analysis showed that Iso rats were overall most sensitive to changes in cocaine unit dose; however, modSPS had no effect on the cocaine dose response curve. Further, there was no effect of either modSPS or differential rearing on expression of glucocorticoid receptor (GR) in hypothalamus, medial prefrontal cortex, amygdala, or nucleus accumbens. By using modSPS in combination with Iso housing, this study identified unique contributions of each stressor to acquisition of cocaine self-administration.
\end{abstract}

\section{Keywords}

cocaine self-administration; single prolonged stress; isolation; environmental enrichment; PTSD

\footnotetext{
"Corresponding author: Rebecca S. Hofford, 741 S. Limestone, 448C BBSRB, University of Kentucky, Lexington KY, 40536, rebecca.hofford@uky.edu.

Publisher's Disclaimer: This is a PDF file of an unedited manuscript that has been accepted for publication. As a service to our customers we are providing this early version of the manuscript. The manuscript will undergo copyediting, typesetting, and review of the resulting proof before it is published in its final citable form. Please note that during the production process errors may be discovered which could affect the content, and all legal disclaimers that apply to the journal pertain.
} 


\section{Introduction}

Anxiety and mood disorders, induced by or precipitated by stress, are often co-morbid with addiction [1]. In fact, studies suggest that lifetime prevalence of addiction is double in people suffering from mood or anxiety disorders compared to the general population [1]. Specifically, post-traumatic stress disorder (PTSD), a disorder typically caused by exposure to a traumatic event and characterized by flashbacks of the event, generalized anxiety, fear extinction retention deficits, and anhedonia [2,3], is $3-10 \%$ more common in substance abusers than in the general public [4]. In addition, one estimate suggests that PTSD is twice as common in individuals with a cocaine use disorder specifically [5].

The high rate of co-morbidity between PTSD and cocaine use disorder is not surprising since stress and addiction engage many of the same underlying brain systems, including the hypothalamic-pituitary-adrenal (HPA) axis [6]. Normal activation of this system, comprised of a series of structures in the central nervous system and periphery, results in release of glucocorticoids from the adrenal glands (primarily cortisol in humans and corticosterone (CORT) in rodents [7]). Glucocorticoids are released during stress and are thus considered "stress hormones". These steroid hormones can cross the blood-brain-barrier and act as negative regulators of their own release by binding glucocorticoid receptors (GRs [8]). Long-lasting perturbations in HPA axis function can have negative health consequences, including impaired immune function [9] and altered glucose metabolism [10].

Patients with PTSD and cocaine use disorder display abnormal HPA axis function, albeit in different ways. PTSD produces lower circulating CORT levels [11] and enhanced negative feedback due to greater GR expression [12,13]. Conversely, acute and repeated psychostimulant use causes enhanced release of cortisol in humans [14] and CORT in rats [15]. CORT is reinforcing by itself [16, 17], but also has been shown to alter psychostimulant self-administration. Release of CORT is linked to cocaine selfadministration, as adrenalectomy drastically attenuates self-administration and flattens the dose-response curve [18, 19]. GRs partially mediate CORT's effects on self-administration; GR antagonists [20-22] and GR knockout in dopamine D1 immunoreactive cells also decrease self-administration of psychostimulants [23].

Another reason PTSD and addiction often co-occur is that stress directly contributes to many stages of the addiction cycle, including escalation from casual use to problematic use and relapse [24]. Rodent models demonstrate that exposure to stress can increase overall levels of cocaine self-administration [25], escalation of intake [26], and drug-seeking [27].

However, not all stressors produce the same behavioral and biological effects. Important factors affecting outcome include number of lifetime stressors [28, 29], age of the individual at first stress exposure [30], and the specific stress protocol employed [31].

Early life stress, which occurs during childhood or adolescence, can predispose individuals to develop PTSD [32] and substance use disorder [33]. Likewise, preclinical experiments produce similar findings [34]. One common model of early life stress involves rearing rats during adolescence in social isolation (isolation condition, Iso). Decades of research have demonstrated that rats reared in Iso during adolescence display profound behavioral 
differences from rats raised in standard conditions (Std) or enriched conditions (Enr). Most studies indicate that Iso rats are more prone to addictive- and depressive-like behavior, while Enr rats are protected from these same outcomes [35-37]. In addition, several studies have suggested that levels of HPA markers such as CORT [21, 38-40] and GR in hippocampus [41-43] differ between rats raised in Iso and Enr, although the data are inconsistent.

In rodents, drug-taking or seeking after stress depends on the stress protocol employed [44, 45]. While the effects of restraint stress and unpredictable foot-shock on drug taking and seeking have been well studied, relatively few studies have employed a stress paradigm relevant to PTSD. One PTSD-like model utilizes a single prolonged stress (SPS), consisting of one episode of a series of 3 stressors: $2 \mathrm{hr}$ restraint stress, followed by $15 \mathrm{~min}$ swim stress, and finished with ether gas exposure until loss of consciousness [46]. This model possesses validity since animals exposed to SPS demonstrate fear extinction retention deficits and enhanced negative feedback of the HPA axis, both common in patients with PTSD $[47,48]$. While initial studies employing the whole SPS model did not observe differences in cocaine self-administration after SPS exposure [49], a modified SPS protocol that substituted isoflurane for ether found stress-induced decreases in cocaine escalation [50]. However, no previous studies have determined if SPS-induced alterations in cocaine self-administration can be moderated by isolation rearing.

Given the literature on early life stress and the behavioral differences between Iso and Enr rats, it is likely that adolescent exposure to these environments could differentially affect cocaine self-administration and HPA response to SPS in adulthood. The current experiments administered 2 stressors: isolation rearing starting in adolescence followed by a modified SPS (modSPS) session in young adulthood. The modSPS session was used in lieu of SPS to avoid ceiling effects on stress responses; differences between rearing environments are most often observed at lower doses of drug in pharmacological studies [20, 37]. This modified protocol has demonstrated similar changes in the HPA axis, but does not reproduce fear extinction retention deficits [48]. To date, no experiments have examined cocaine selfadministration after exposure to this modified procedure. Analysis of acquisition, maintenance, and the dose-response curve of cocaine self-administration were conducted and changes in CORT and GR expression were quantified. It was hypothesized that Iso rats would be most sensitive to the effects of modSPS; they were expected to acquire cocaine self-administration faster than other groups and were expected to show greater CORT release and a greater increase in GR expression in brain following modSPS. In testing this hypothesis, we chose to use a relatively high unit dose of cocaine $(0.56 \mathrm{mg} / \mathrm{kg} / \mathrm{infusion})$ in order to minimize baseline (non-stressed) differences in Enr and Iso rats in rates of cocaine self-administration [37]. Thus, we expected to observe differences in cocaine intake among Enr, Std, and Iso rats, but only when exposed to modSPS.

\section{Material and Methods}

\subsection{Animals and Housing}

Male Sprague-Dawley rats purchased from envigo-Harlan (Indianapolis, IN) arrived in the colony PND 21-22 and were immediately assigned to either an Enr, a Std or an Iso environment where they remained for the entire experiment. Enr rats were housed 5-12 per 
cage in large, stainless steel cages $(122 \times 61 \times 45.5 \mathrm{~cm})$ with ample bedding. Fourteen objects were placed throughout the cage and were rearranged daily and replaced every other day. Std rats were pair housed in standard IVC cages $(33 \times 38 \times 20 \mathrm{~cm})$ with bedding but no objects and Iso rats were housed singly in small stainless steel cages $(17 \times 24 \times 20 \mathrm{~cm})$ with wire mesh bottoms and no objects. All rats were kept in a humidity and temperaturecontrolled room on a 12-hr light dark cycle (lights on at 7:00 AM). Food and water were available continuously in the home cage environment. All procedures were approved by the University of Kentucky Animal Care and Use Committee and conformed to NIH standards.

\subsection{Surgical Procedures}

Between PND 55 and 60, all rats received jugular catheter implantation surgery. Briefly, rats were anesthetized with a ketamine (Butler Schein, Dublin OH)/xylazine (Akorn, Inc., Decatur IL)/acepromazine (Boehringer Ingelheim, St. Joseph MO) cocktail (75/7.5/0.75 $\mathrm{mg} / \mathrm{kg} ; 0.15 \mathrm{ml} / 100 \mathrm{~g}$ body weight; i.p.). A jugular catheter was implanted into the right jugular vein, threaded under the skin, and exited the body through an incision on the scalp. The catheter port was attached to the skull using four jeweler's screws and dental acrylic. Rats were allowed to recover for one week before experimental procedures began. During this time, rats received a $5.5 \mathrm{mg} / \mathrm{kg}$ carprofen injection (s.c.) on the day following surgery and daily catheter infusions of $0.2 \mathrm{ml}$ gentamicin in sterile saline $(10.15 \mathrm{mg} / \mathrm{ml}$, Abraxis BioScience, Los Angeles CA) followed by $0.2 \mathrm{ml}$ of post-flush solution [containing $1 \%$ gentamicin (10.15 mg/ml) and 3\% heparin (1000 USP units/ml, Abraxis BioScience, Los Angeles CA) in sterile saline] from recovery days 2-7.

\subsection{Modified Single Prolonged Stress (modSPS)}

At 7-8 days after surgery, one half of the rats from each rearing condition underwent a modSPS protocol [48]. Stressed (modSPS) rats were restrained in plastic restrainers for $2 \mathrm{hr}$, followed immediately by placement in $24-26^{\circ} \mathrm{C}$ water for a maximum of 15 min under constant surveillance. After this, rats were removed from the water, lightly dried, and placed in individual empty cages on heating pads for $1 \mathrm{hr}$ before being returned to their respective housing environments. The other half of the rats from each rearing condition remained in the same testing room but were not subjected to stress, thus serving as non-stressed controls (control). ModSPS and control rats were housed together in the Enr cage and some of the Std cages. Other Std cages contained 2 modSPS rats or 2 control rats.

\subsection{Cocaine self-administration}

2.4.1 Apparatus-All self-administration sessions were conducted in standard 2-lever operant conditioning chambers $(28 \times 24 \times 21 \mathrm{~cm}$; ENV-008CT; MED Associates, St. Albans VT) equipped with syringe pumps for drug delivery (PHM-100; MED Associates). Located above each retractable lever was a white cue light.

2.4.2 Acquisition-Seven days following modSPS, rats in the cocaine self-administration study ( $\mathrm{n}=31$ total; $\mathrm{n}=4-6$ rats/group) were trained to self-administer $0.56 \mathrm{mg} / \mathrm{kg} / \mathrm{infusion}$ cocaine on a FR1 schedule using an autoshaping procedure. A 7 day incubation after SPS or modSPS is sufficient to produce changes in HPA axis activity reminiscent of PTSD, while shorter time points are not [51]. The autoshaping procedure lasted for 7 days. During 
autoshaping sessions, the active lever was inserted into the chamber on a VI90 sec schedule with a $15 \mathrm{sec}$ limited hold. Once presented, the active lever remained extended for $15 \mathrm{sec}$, after which the lever was retracted and $0.56 \mathrm{mg} / \mathrm{kg}$ infusion cocaine was immediately delivered. However, if a lever press was made within $15 \mathrm{sec}$ of the lever presentation, the lever would retract and a $0.56 \mathrm{mg} / \mathrm{kg}$ infusion of cocaine would be immediately delivered. Rats received a total of 10 infusions of cocaine during each autoshaping session within a 15min window. Autoshaping sessions lasted for a total of $1 \mathrm{hr}$. Additionally, the inactive lever was present during the entire autoshaping session, where any lever press made had no consequence. After the end of autoshaping, all rats were returned to their respective housing conditions for $1 \mathrm{hr}$ and then were returned to the operant boxes where they were allowed to self-administer $0.56 \mathrm{mg} / \mathrm{kg} /$ infusion cocaine ( $5.9 \mathrm{sec}$ infusion duration) on a FR1 (20-sec signaled time out) for $1 \mathrm{hr}$. The signal consisted of illumination of the cue light above the active lever (counterbalanced) for $20 \mathrm{sec}$. Responses on the inactive lever produced no programmed consequence. At the end of each self-administration session, rats received 0.2 $\mathrm{ml}$ of post-flush solution.

2.4.3 Maintenance-After 7 days, rats continued their daily 60-min sessions without autoshaping until the mean number of active lever presses within each group was stable $(<$ $20 \%$ variability over 3 consecutive days).

\subsubsection{Dose-Response Curve Assessment and Verification of Catheter Patency}

-Upon reaching stability on the training dose, the unit dose of cocaine was decreased to $0.32 \mathrm{mg} / \mathrm{kg} / \mathrm{infusion}$ for 3 days, followed by $0.18 \mathrm{mg} / \mathrm{kg} / \mathrm{infusion}$ for 3 days. One day following the last self-administration session, rats were given a $15 \mathrm{mg} / \mathrm{kg}$ morphine bolus i.v.; lack of catatonia within 3 secs of infusion was considered lack of catheter patency. Data from rats that lost headmounts or had leaking headmounts were thrown out of the entire experiment. In total, 5 rats were excluded due to headmount compromise; all remaining rats passed patency check.

\subsection{Tissue Collection}

In a separate group of rats ( $\mathrm{n}=41$ total; $\mathrm{n}=5-9$ rats/group), blood was drawn from the jugular catheter of modSPS and control rats 7 days following catheter surgery. These rats did not undergo self-administration procedures but received jugular catheter surgery and recovery as described above. In modSPS rats, approximately $0.2 \mathrm{ml}$ of blood was drawn $1 \mathrm{hr}$ before stress (baseline, time 1), immediately following stress (time 2), and $1 \mathrm{hr}$ following the end of stress (time 3); equivalent time points were used for control rats. It should be noted that blood samples were not successfully drawn for every rat at every time point due to catheter patency difficulties. However, all rats successfully had blood drawn at least once. Seven days following modSPS, rats were rapidly decapitated and hypothalamus (Hypo), medial prefrontal cortex (mPFC), amygdala (Amyg) and nucleus accumbens (NAc) were removed and immediately frozen on dry ice. A 7 day incubation after SPS or modSPS is sufficient to produce changes in HPA axis activity reminiscent of PTSD, while shorter time points are not [51]. Data is not included for one Amyg, one mPFC and one NAc due to failure in brain dissection and collection. 


\subsection{CORT levels}

Plasma was collected after blood centrifugation at 3,000 $g$ for $15 \mathrm{~min}$. Plasma was diluted 1:500 for corticosterone ELISA (Cayman Chemical \#501320, Ann Arbor MI) following assay instructions. Samples were run in duplicate and absorbance was read at $405 \mathrm{~nm}$ after 1 $\mathrm{hr}$ incubation with provided Ellman's solution. Since group means did not significantly differ between modSPS and control rats at time 1, modSPS and control CORT levels within each environment were combined. Percent CORT at times 1, 2, and 3 were calculated using the equation: (mean group CORT time 1, 2, or 3/mean group CORT time 1) $\times 100$.

\subsection{Western blot for GR}

Lysis buffer (1:100 Halt protease/phosphate inhibitor-ThermoFisher Scientific, Pittsburgh PA and 1:1000 PMSF/EtOH in RIPA buffer-ThermoFisher Scientific, Pittsburgh PA) was added in a volume of $300 \mu \mathrm{l}$ to each sample and homogenized with a Teflon pestle. Samples were centrifuged at $23,000 \mathrm{~g}$ for $20 \mathrm{~min}$ and supernatant was collected. Levels of total protein were assessed using a BCA protein assay kit per assay instructions (ThermoFisher Scientific, Pittsburgh PA). $\beta$-mercaptoethanol was added to $30 \mu \mathrm{g}$ protein from samples and boiled for $15 \mathrm{~min}$ at $65^{\circ} \mathrm{C}$. Ten $\mu \mathrm{l}$ of each prepared sample was loaded on a 4-12\% MiniPROTEAN SDS-polyacrylamide gel (Bio-Rad, Hercules CA) concurrent with a protein standard (Bio-Rad, Hercules CA). Because there were 6 groups of rats with unequal sample sizes, 1 or 2 animals from each group were represented on every gel, ensuring that relatively equal number of rats from each environment and each stress group were represented. Proteins were separated by electrophoresis using a Bio-Rad PowerPac HC Mini-PROTEAN TetraSystem at $95 \mathrm{~V}$. Proteins were transferred to a nitrocellulose membrane (Bio-Rad, Hercules CA) at $100 \mathrm{~V}$ for $60 \mathrm{~min}$. Blots were blocked with $5 \%$ nonfat milk (w/v) in $1 \mathrm{x}$ PBS for 60 min. Primary antibody for GR (ab3580, abcam, Cambridge MA, 1:500) and GAPDH (ab8245, abcam, Cambridge MA, 1:1000) were added to 5\% nonfat milk (w/v) in PBS-Tween 20 and was incubated at $4{ }^{\circ} \mathrm{C}$ overnight with gentle agitation. On the following day, secondary antibodies (anti-mouse: Rockland IRDye 800CW, 1:5000; anti-rabbit: LifeTechnologies AlexaFluor 680, 1:5000) were added to 5\% nonfat milk (w/v) in PBSTween 20 for $60 \mathrm{~min}$, and optical density was determined using an Odyssey Infrared Imager (LI-COR, Lincoln NE). Amount of GR was normalized to the GAPDH loading control using the formula: GR optical density/GAPDH optical density. Normalized values were converted to percentage of control Std for graphing purposes.

\subsection{Statistical Analysis}

For the cocaine self-administration study, active lever presses during acquisition, maintenance, and dose-response were analyzed using separate linear mixed effect models with subject as a random factor and environment, stress group, and session or dose as fixed factors. Linear regression was also used to compare the slope of the acquisition line between modSPS and control rats within each environment. Total CORT at time 1 was analyzed using a Kruskal-Wallis with Dunn's multiple comparison test and percent CORT at times 1, 2 , and 3 were analyzed using a linear mixed effect model with subject as a random factor and environment, stress group, and time point as fixed factors. Linear mixed effects (LME) was used for these analyses because cocaine self-administration data and CORT data 
contained missing values, for which LME is most appropriate. Additionally, LME allows for random factors into the statistical design, thereby increasing power and reducing Type I error [52]. Because there were no continuous independent variables, normalized GR protein levels were subjected to separate $2 \times 3$ between-subjects ANOVAs for each brain region. Significant interactions were probed using Tukey's HSD test. Cage composition of Std cages (whether there were 2 modSPS, 2 control, or 1 modSPS and 1 control), did not affect any measured outcome. Therefore, cage composition was not included as a factor in any analysis.

\section{Results}

\subsection{Cocaine self-administration}

For acquisition (Fig 1), linear mixed effects identified main effects of stress $(F(1,25)=$ $10.05, p<0.05)$ and session $(F(1,25)=11.11, p<0.05)$ on active lever presses. These main effects indicated that stress produced an overall reduction in lever pressing and that lever pressing increased across sessions. Further analyses within each environment revealed that the slope of the acquisition line differed significantly between modSPS and control groups within the Iso environment only $(F(1,73)=4.96, p<0.05)$. Iso rats in the modSPS condition showed little responding early in training, but rapidly acquired self-administration to reach the same level of lever pressing as control rats by the end of acquisition training. In contrast, modSPS and control rats in the Enr and Std groups acquired cocaine self-administration at the same gradual rate despite modSPS groups' lower overall intake.

For maintenance (Fig 2), linear mixed effects revealed no significant main effects or interactions, indicating that rates of lever pressing were similar across treatment groups during this phase.

For dose-response analysis (Fig 3), linear mixed effects revealed a significant main effect of dose $(F(2,50)=33.03, p<0.05)$ and an interaction between environment and dose $(F(4,50)$ $=3.55, p<0.05$ ). Tukey's HSD post hoc analysis revealed that Std rats exhibited a flat doseresponse curve, i.e., there was no difference between doses within the Std group. In contrast, Enr rats demonstrated a significant increase in active lever presses between the highest $(0.56$ $\mathrm{mg} / \mathrm{kg}$ ) and lowest $(0.18 \mathrm{mg} / \mathrm{kg})$ doses only ( $p<0.05)$. In addition, Iso rats demonstrated a significant increase in active lever presses between the lowest and highest doses $(p<0.05)$, as well as between the intermediate dose $(0.32 \mathrm{mg} / \mathrm{kg})$ and the lowest dose $(0.18 \mathrm{mg} / \mathrm{kg} ; p<$ 0.05 ), indicating that among all 3 rearing conditions, Iso rats were most sensitive to dose adjustments.

\subsection{CORT levels}

Sample sizes for CORT analysis were not equal and data were not normally distributed; therefore, Kruskal-Wallis was used in lieu of classic ANOVA. At baseline (time 1), KruskalWallis test identified a significant difference between groups $(H=6.44, p<0.05)$. Dunn's comparison identified a significant difference between Std and Iso CORT levels, with Iso expressing greater amounts of CORT than Std (Fig 4A). For the other time points (Fig 4B), linear mixed effects identified main effects of stress $(F(1,29.13)=10.60, p<0.05)$ and time 
point $(F(1,25.84)=7.90, p<0.05)$. Additionally, there were significant environment $\mathrm{x}$ time point $(F(2,25.40)=4.10, p<0.05)$ and stress $\mathrm{x}$ time point $(F(1,25.84)=4.34, p<0.05)$ interactions. Tukey's post hoc analysis revealed that Std rats had greater percent baseline CORT than Iso rats at time point 3. For modSPS rats, percent baseline CORT at time 1 was significantly lower than percent baseline CORT at times 2 and 3, but percent baseline CORT did not differ between time points 2 and 3. Finally, modSPS rats had greater percent baseline CORT than control rats at time 2 only (all analyses $p<0.05$ ).

\subsection{Western blot for GR}

Residuals from Amyg and mPFC were not normally distributed and thus data for these two brain regions were log transformed before analysis. No significant differences in GR expression were identified between groups in any brain region tested (Fig 5).

\section{Discussion}

One important finding from this study was that modSPS produced an overall decrease in cocaine self-administration during initial acquisition. However, contrary to our hypothesis, rearing environment largely did not influence cocaine self-administration or HPA markers after modSPS. One exception to this was the finding that the pattern of acquisition in modSPS Iso rats differed from modSPS Enr and Std rats which was represented by a steeper acquisition slope. While all modSPS groups displayed a low rate of lever pressing at the beginning of training, modSPS Iso rats displayed a greater acceleration of responding across sessions compared to both modSPS Std and modSPS Enr rats. One possible reason there was an effect of modSPS only in Iso rats could be that stressed rats in the other two groups (Enr and Std) were housed with control rats, which may have ameliorated the effect of modSPS in those groups.

While previous studies have shown that Iso rats can acquire cocaine self-administration more rapidly than standard-housed or group-housed rats [53, 54], this effect was not present in the current control groups. This latter result is likely due to the high unit dose of cocaine used in the current study $(0.56 \mathrm{mg} / \mathrm{kg} /$ infusion $)$, as the effect of differential rearing tends to occur only at low unit doses [36, 37].

During acquisition, modSPS rats self-administered significantly less cocaine than control rats regardless of environment. Studies using other versions of SPS did not observe any differences in acquisition of cocaine self-administration [49, 50]. However, several methodological differences exist between prior experiments and the current study, including the stress protocol used, timing of catheter surgery, training dose, and training procedure. To our knowledge, this is the first study to use modSPS (specifically, $2 \mathrm{hr}$ restraint followed by 15 mins cold swim) to measure stress-induced changes in cocaine self-administration. It is possible that this iteration of SPS produces differences in cocaine intake during acquisition, whereas full SPS and SPS with substituted isoflurane do not. Additionally, the current study used a relatively high training dose $(0.56 \mathrm{mg} / \mathrm{kg} /$ infusion $)$ to minimize baseline differences in intake between control Enr and Iso rats [20,37], whereas previous studies used doses between 0.1 and $0.3 \mathrm{mg} / \mathrm{kg} / \mathrm{infusion}$. Finally, the current study trained rats using an autoshaping procedure in which rats received 10 non-contingent infusions $1 \mathrm{hr}$ before the 
start of their FR self-administration session. Interestingly, the attenuation of lever pressing due to modSPS only occurred during the acquisition phase (i.e. during the sessions preceded by autoshaping), suggesting that this methodological difference is critical to the modSPSinduced reduction in cocaine intake during acquisition.

Based on previous literature, it is not clear if exposure to SPS or modSPS increases or decreases sensitivity to the reinforcing effects of psychostimulants. For example, one report found that rats exposed to SPS were more sensitive to methamphetamine's locomotor stimulating properties but less sensitive to stereotyped behavior elicited by methamphetamine [55]. Another study found that rats exposed to SPS consumed less sucrose, had reduced cocaine CPP, and self-administered significantly less cocaine during long-access conditions [50], suggesting that SPS reduces reward sensitivity. The current results are most consistent with these latter findings suggesting that modSPS decreases reward sensitivity. Interestingly, anhedonia is a major symptom of PTSD, making this interpretation appealing from a translational perspective.

ModSPS did not affect any other behavioral outcomes during maintenance and doseresponse assessment. As stated previously, this might be explained by the absence of autoshaping during these stages. Alternatively, it is possible that the effects of modSPS on cocaine self-administration are transient. In one study using full SPS, some behavioral effects of SPS dissipated within 14 days [56], while other studies found that effects of SPS on cocaine self-administration lasted beyond 14 days $[49,50]$. The current study lasted $\sim 40$ days total, but autoshaping occurred between days 1 and 7 .

During the dose-response phase, regardless of the stress treatment, there was a significant environment $x$ dose interaction. While all doses tested were on the descending limb of the normal dose-response curve [37], only Enr and Iso rats showed a significant dose-dependent increase in active lever presses as the dose was lowered. Importantly, there was evidence that Iso rats were most sensitive to cocaine reinforcement because they were the only group to significantly increase their active lever presses when the dose was reduced from 0.32 to 0.18 $\mathrm{mg} / \mathrm{kg}$ /infusion. As reported previously [37], Iso rats typically self-administer more cocaine than Enr rats at low unit doses, although this effect is minimized when Enr and Iso rats are trained at higher cocaine doses.

Regardless of differential rearing, modSPS increased CORT release immediately after stress and these levels remained high $1 \mathrm{hr}$ after stress, indicating that the modified procedure produced acute HPA axis activity similar to full SPS [46]. Basal levels of CORT (prior to stress) were elevated in Iso rats compared to Std rats. The elevated basal CORT in Iso rats may reflect an effect of handling, as Iso rats did not get handled until immediately prior to surgery. Thus, it is possible that increased basal CORT in Iso rats reflects a slight stress response to novel handling. Interestingly, however, basal CORT response did not differ between Enr and Iso groups, despite a clear trend for Iso rats to have greater CORT than Enr. There is current debate about whether Enr or Iso groups are more 'stressed' [57, 58]. Both social isolation (experienced by Iso rats) and exposure to novelty (experienced by Enr rats) can induce CORT release and other physiological signs of stress. The effects of differential housing on CORT seems dependent on the exact sampling procedure and housing paradigm, 
with some studies reporting highest basal CORT in Iso rats [21, 38], and others reporting highest basal CORT in Enr or Std rats [39, 40]. While increased levels of CORT sometimes indicates distress, elevated CORT levels, especially in response to novelty, can be adaptive and might be beneficial to individuals over time [57, 59]. This suggests that baseline CORT levels of Enr, Std, and Iso rats might not be meaningful predictors of behavioral or physiological outcomes. Hence, care should be taken when interpreting the observation that baseline CORT levels did not differ between Enr and Iso rats. Similarly, the pattern of CORT activation appears to be different across rearing environments. For instance, Enr and Iso rats exposed to modSPS had peak CORT at time 2, whereas Std rats had highest CORT at time 3. This suggests either that Std rats had a delayed CORT response to stress or that Enr and Iso rats were better able to recover their CORT response to resting levels.

Finally, no differences in GR were found in any brain region tested between environments or stress groups. This contrasts with a previous study showing greater GR in prefrontal cortex (PFC) of rats exposed to full SPS [48]; however, several differences in methodology (as discussed above) could explain discrepancies between those and the current study. Most notably, the previous experiment used full SPS which produces greater increases in GR expression in hippocampus and PFC compared to the modSPS protocol, and the previous study measured GR in the entire PFC, while this study quantified GR in mPFC [48]. The current study could have been improved by measuring GR in hippocampus, thus allowing for better comparison to previous SPS literature. However, the current results are consistent with a different report showing that Enr and Iso control rats do not express different levels of GR in mPFC, Amyg or NAc [20].

The fact that modSPS decreased cocaine intake early in training without affecting any HPA axis outcome measured suggests that modSPS reduced cocaine intake via a neural system independent of the HPA axis. In addition to HPA systems, evidence suggests that exposure to SPS directly alters dopamine reuptake by up-regulation of the dopamine transporter and down-regulation of D2 receptors in NAc [50], suggesting at least one alternative mechanism for the decrease in cocaine self-administration observed here.

Given the unexpected findings that modSPS reduced cocaine self-administration early in training and produced no alterations in prefrontal GR, it is also possible that modSPS is not an appropriate model of PTSD. This is consistent with the finding that SPS, but not modSPS, produces extinction retention deficits [48].

\section{Conclusions}

The current experiment identified unique alterations in the acquisition of cocaine selfadministration using rearing environment and a rodent model of PTSD. The importance of stress history on PTSD outcomes is often overlooked. While rearing environment did not alter most effects of modSPS on cocaine self-administration, the effect of other stressors during the juvenile or adolescent period might impact these outcomes. Experiments using the SPS model in combination with other stressors are necessary for successful development of pharmacotherapies for co-morbid PTSD and substance use disorder. 


\section{Acknowledgments}

This work was supported by National Institute of Health grants [DA012964, DA036291].

\section{References}

1. Conway KP, Compton W, Stinson FS, Grant BF. Lifetime comorbidity of DSM-IV mood and anxiety disorders and specific drug use disorders: results from the National Epidemiologic Survey on Alcohol and Related Conditions. J Clin Psychiatry. 2006; 67:247-57. [PubMed: 16566620]

2. APA. Diagnostic and statistical manual of mental disorders. 5. Arlington, VA: American Psychiatric Publishing; 2003.

3. Nawijn L, van Zuiden M, Frijling JL, Koch SBJ, Veltman DJ, Olff M. Reward functioning in PTSD: A systematic review exploring the mechanisms underlying anhedonia. Neuroscience \& Biobehavioral Reviews. 2015; 51:189-204. [PubMed: 25639225]

4. Cottler LB, Compton WM, Mager D, Spitznagel EL, Janca A. Posttraumatic stress disorder among substance users from the general population. American Journal of Psychiatry. 1992; 149:664-70. [PubMed: 1575258]

5. Saunders EC, Lambert-Harris C, McGovern MP, Meier A, Xie H. The Prevalence of Posttraumatic Stress Disorder Symptoms among Addiction Treatment Patients with Cocaine Use Disorders. Journal of psychoactive drugs. 2015; 47:42-50. [PubMed: 25715071]

6. Koob G, Kreek MJ. Stress, Dysregulation of Drug Reward Pathways, and the Transition to Drug Dependence. The American journal of psychiatry. 2007; 164:1149-59. [PubMed: 17671276]

7. Blackwell RE, Guillemin R. Hypothalamic control of adenohypophysial secretions. Annu Rev Physiol. 1973; 35:357-90. [PubMed: 4350616]

8. Ratka A, Sutanto W, Bloemers M, de Kloet ER. On the role of brain mineralocorticoid (type I) and glucocorticoid (type II) receptors in neuroendocrine regulation. Neuroendocrinology. 1989; 50:11723. [PubMed: 2550833]

9. Garvy BA, Fraker PJ. Suppression of the antigenic response of murine bone marrow B cells by physiological concentrations of glucocorticoids. Immunology. 1991; 74:519-23. [PubMed: 1769699]

10. Khani S, Tayek JA. Cortisol increases gluconeogenesis in humans: its role in the metabolic syndrome. Clinical Science. 2001; 101:739. [PubMed: 11724664]

11. Yehuda R, Teicher MH, Trestman RL, Levengood RA, Siever LJ. Cortisol regulation in posttraumatic stress disorder and major depression: A chronobiological analysis. Biological Psychiatry. 1996; 40:79-88. [PubMed: 8793040]

12. Yehuda R, Boisoneau D, Mason JW, Giller EL. Glucocorticoid receptor number and cortisol excretion in mood, anxiety, and psychotic disorders. Biological Psychiatry. 1993; 34:18-25. [PubMed: 8373936]

13. Yehuda R, Southwick SM, Krystal JH, Bremner D, Charney DS, Mason JW. Enhanced suppression of cortisol following dexamethasone administration in posttraumatic stress disorder. American Journal of Psychiatry. 1993; 150:83-6. [PubMed: 8417586]

14. Baumann MH, Gendron TM, Becketts KM, Henningfield JE, Gorelick DA, Rothman RB. Effects of intravenous cocaine on plasma cortisol and prolactin in human cocaine abusers. Biological Psychiatry. 1995; 38:751-5. [PubMed: 8580229]

15. Mantsch JR, Schlussman SD, Ho A, Kreek MJ. Effects of Cocaine Self-Administration on Plasma Corticosterone and Prolactin in Rats. Journal of Pharmacology and Experimental Therapeutics. 2000; 294:239. [PubMed: 10871318]

16. Deroche, Vr, Piazza, PV., Deminière, J-M., Moal, ML., Simon, H. Rats orally self-administer corticosterone. Brain Research. 1993; 622:315-20. [PubMed: 8242374]

17. Piazza PV, Deroche V, Deminière JM, Maccari S, Le Moal M, Simon H. Corticosterone in the range of stress-induced levels possesses reinforcing properties: implications for sensation-seeking behaviors. Proceedings of the National Academy of Sciences of the United States of America. 1993; 90:11738-42. [PubMed: 8265619] 
18. Goeders NE, Guerin GF. Effects of surgical and pharmacological adrenalectomy on the initiation and maintenance of intravenous cocaine self-administration in rats. Brain Research. 1996; 722:145-52. [PubMed: 8813360]

19. Deroche V, Marinelli M, Le Moal M, Piazza PV. Glucocorticoids and Behavioral Effects of Psychostimulants. II: Cocaine Intravenous Self-administration and Reinstatement Depend on Glucocorticoid Levels. Journal of Pharmacology and Experimental Therapeutics. 1997; 281:14017. [PubMed: 9190876]

20. Hofford RS, Prendergast MA, Bardo MT. Pharmacological manipulation of glucocorticoid receptors differentially affects cocaine self-administration in environmentally enriched and isolated rats. Behavioural brain research. 2015; 283:196-202. [PubMed: 25655510]

21. Stairs DJ, Prendergast MA, Bardo MT. Environmental-induced differences in corticosterone and glucocorticoid receptor blockade of amphetamine self-administration in rats. Psychopharmacology. 2011; 218:293. [PubMed: 21887496]

22. Fiancette J-F, Balado E, Piazza P-V, Deroche-Gamonet V. Mifepristone and spironolactone differently alter cocaine intravenous self-administration and cocaine-induced locomotion in C57BL/6J mice. Addiction Biology. 2010; 15:81-7. [PubMed: 19799583]

23. Ambroggi F, Turiault M, Milet A, Deroche-Gamonet V, Parnaudeau S, Balado E, et al. Stress and addiction: glucocorticoid receptor in dopaminoceptive neurons facilitates cocaine seeking. Nat Neurosci. 2009; 12:247-9. [PubMed: 19234455]

24. Sinha R. How does stress increase risk of drug abuse and relapse? Psychopharmacology. 2001; 158:343-59. [PubMed: 11797055]

25. Miczek KA, Mutschler NH, Mizcek KA. Activational effects of social stress on IV cocaine selfadministration in rats. Psychopharmacology. 1996; 128:256-64. [PubMed: 8972545]

26. Boyson CO, Holly EN, Shimamoto A, Albrechet-Souza L, Weiner LA, DeBold JF, et al. Social Stress and CRF-Dopamine Interactions in the VTA: Role in Long-Term Escalation of Cocaine Self-Administration. The Journal of Neuroscience. 2014; 34:6659. [PubMed: 24806691]

27. Erb S, Shaham Y, Stewart J. Stress reinstates cocaine-seeking behavior after prolonged extinction and a drug-free period. Psychopharmacology. 1996; 128:408. [PubMed: 8986011]

28. Scott ST. Multiple Traumatic Experiences and the Development of Posttraumatic Stress Disorder. Journal of Interpersonal Violence. 2007; 22:932-8. [PubMed: 17575070]

29. Mahoney JJ Iii, Newton TF, Omar Y, Ross EL, De La Garza R Ii. The relationship between lifetime stress and addiction severity in cocaine-dependent participants. European Neuropsychopharmacology. 2013; 23:351-7. [PubMed: 22748418]

30. Ricon T, Toth E, Leshem M, Braun K, Richter-Levin G. Unpredictable chronic stress in juvenile or adult rats has opposite effects, respectively, promoting and impairing resilience. Stress: The International Journal on the Biology of Stress. 2012; 15:11-20.

31. Bowers SL, Bilbo SD, Dhabhar FS, Nelson RJ. Stressor-specific alterations in corticosterone and immune responses in mice. Brain, Behavior, and Immunity. 2008; 22:105-13.

32. Lang AJ, Aarons GA, Gearity J, Laffaye C, Satz L, Dresselhaus TR, et al. Direct and indirect links between childhood maltreatment, posttraumatic stress disorder, and women's health. Behavioral Medicine. 2008; 33:125-36. [PubMed: 18316270]

33. Alvarez-Alonso MJ, Jurado-Barba R, Martinez-Martin N, Espin-Jaime JC, Bolaños-Porrero C, Ordoñez-Franco A, et al. Association between maltreatment and polydrug use among adolescents. Child Abuse \& Neglect. 2016; 51:379-89. [PubMed: 26318780]

34. Lewis CR, Staudinger K, Tomek SE, Hernandez R, Manning T, Olive MF. Early life stress and chronic variable stress in adulthood interact to influence methamphetamine self-administration in male rats. Behav Pharmacol. 2016; 27:182-4. [PubMed: 26176409]

35. Alvers KM, Marusich JA, Gipson CD, Beckmann JS, Bardo MT. Environmental enrichment during development decreases intravenous self-administration of methylphenidate at low unit doses in rats. Behav Pharmacol. 2012; 23:650-7. [PubMed: 22914073]

36. Bardo MT, Klebaur JE, Valone JM, Deaton C. Environmental enrichment decreases intravenous self-administration of amphetamine in female and male rats. Psychopharmacology. 2001; 155:278. [PubMed: 11432690] 
37. Green TA, Alibhai IN, Roybal CN, Winstanley CA, Theobald DEH, Birnbaum SG, et al. Environmental Enrichment Produces a Behavioral Phenotype Mediated by Low Cyclic Adenosine Monophosphate Response Element Binding (CREB) Activity in the Nucleus Accumbens. Biological psychiatry. 2010; 67:28-35. [PubMed: 19709647]

38. Dronjak S, Gavrilović L, Filipović D, Radojčić MB. Immobilization and cold stress affect sympatho-adrenomedullary system and pituitary-adrenocortical axis of rats exposed to long-term isolation and crowding. Physiology \& Behavior. 2004; 81:409-15. [PubMed: 15135012]

39. Raz S. Ameliorative effects of brief daily periods of social interaction on isolation-induced behavioral and hormonal alterations. Physiology \& Behavior. 2013; 116-117:13-22.

40. Konkle ATM, Kentner AC, Baker SL, Stewart A, Bielajew C. Environmental-Enrichment-Related Variations in Behavioral, Biochemical, and Physiologic Responses of Sprague-Dawley and Long Evans Rats. Journal of the American Association for Laboratory Animal Science: JAALAS. 2010; 49:427-36. [PubMed: 20819388]

41. Sampedro-Piquero P, Begega A, Arias JL. Increase of glucocorticoid receptor expression after environmental enrichment: Relations to spatial memory, exploration and anxiety-related behaviors. Physiology \& Behavior. 2014; 129:118-29. [PubMed: 24582669]

42. Olsson T, Mohammed AH, Donaldson LF, Henriksson BG, Seckl JR. Glucocorticoid receptor and NGFI-A gene expression are induced in the hippocampus after environmental enrichment in adult rats. Brain Res Mol Brain Res. 1994; 23:349-53. [PubMed: 8090075]

43. Lin E-JD, Choi E, Liu X, Martin A, During MJ. Environmental enrichment exerts sex-specific effects on emotionality in C57BL/6J mice. Behavioural Brain Research. 2011; 216:349-57. [PubMed: 20732356]

44. Martin-Fardon R, Ciccocioppo R, Massi M, Weiss F. Nociceptin prevents stress-induced ethanolbut not cocaine-seeking behavior in rats. Neuroreport. 2000; 11:1939-43. [PubMed: 10884047]

45. Mantsch JR, Baker DA, Funk D, Lê AD, Shaham Y. Stress-Induced Reinstatement of Drug Seeking: 20 Years of Progress. Neuropsychopharmacology. 2016; 41:335-56. [PubMed: 25976297]

46. Liberzon I, Krstov M, Young EA. Stress-restress: Effects on ACTH and fast feedback. Psychoneuroendocrinology. 1997; 22:443-53. [PubMed: 9364622]

47. Knox D, George SA, Fitzpatrick CJ, Rabinak CA, Maren S, Liberzon I. Single prolonged stress disrupts retention of extinguished fear in rats. Learning \& Memory. 2012; 19:43-9. [PubMed: 22240323]

48. Knox D, Nault T, Henderson C, Liberzon I. Glucocorticoid receptors and extinction retention deficits in the single prolonged stress model. Neuroscience. 2012; 223:163-73. [PubMed: 22863672]

49. Eagle AL, Singh R, Kohler RJ, Friedman AL, Liebowitz CP, Galloway MP, et al. Single prolonged stress effects on sensitization to cocaine and cocaine self-administration in rats. Behavioural Brain Research. 2015; 284:218-24. [PubMed: 25712697]

50. Enman NM, Arthur K, Ward SJ, Perrine SA, Unterwald EM. Anhedonia, Reduced Cocaine Reward, and Dopamine Dysfunction in a Rat Model of Posttraumatic Stress Disorder. Biological Psychiatry. 2015; 78:871-9. [PubMed: 26115790]

51. Liberzon, López, Flagel, Vázquez, Young. Differential Regulation of Hippocampal Glucocorticoid Receptors mRNA and Fast Feedback: Relevance to Post-Traumatic Stress Disorder. Journal of Neuroendocrinology. 1999; 11:11-7. [PubMed: 9918224]

52. Gelman, A., Hill, J. Data analysis using regression and multilevel/hierarchical models. New York: Cambridge University Press; 2006.

53. Puhl MD, Blum JS, Acosta-Torres S, Grigson PS. Environmental Enrichment Protects Against the Acquisition of Cocaine Self-Administration in Adult Male Rats, but Does Not Eliminate Avoidance of a Drug-Associated Saccharin Cue. Behavioural pharmacology. 2012; 23:43-53. [PubMed: 22157144]

54. Baarendse PJJ, Limpens JHW, Vanderschuren LJMJ. Disrupted social development enhances the motivation for cocaine in rats. Psychopharmacology. 2014; 231:1695-704. [PubMed: 24311358] 
55. Eagle AL, Perrine SA. Methamphetamine-induced behavioral sensitization in a rodent model of posttraumatic stress disorder. Drug and Alcohol Dependence. 2013; 131:36-43. [PubMed: 23664123]

56. Wu Z, Tian Q, Li F, Gao J, Liu Y, Mao M, et al. Behavioral changes over time in post-traumatic stress disorder: Insights from a rat model of single prolonged stress. Behavioural Processes. 2016; 124:123-9. [PubMed: 26772783]

57. Crofton EJ, Zhang Y, Green TA. Inoculation stress hypothesis of environmental enrichment. Neuroscience \& Biobehavioral Reviews. 2015; 49:19-31. [PubMed: 25449533]

58. Solinas M, Thiriet N, Chauvet C, Jaber M. Prevention and treatment of drug addiction by environmental enrichment. Progress in Neurobiology. 2010; 92:572-92. [PubMed: 20713127]

59. Liu RT. A developmentally informed perspective on the relation between stress and psychopathology: When the problem with stress is that there is not enough. Journal of abnormal psychology. 2015; 124:80-92. [PubMed: 25688435] 


\section{Highlights}

- Enriched, standard, and isolated rats underwent modified single prolonged stress

- $\quad$ Exposure to modSPS attenuated cocaine self-administration early in training

- Corticosterone increased after modSPS but was not moderated by environment

- $\operatorname{modSPS}$ did not alter glucocorticoid receptor expression in hypo, mPFC, amyg, or NAc 

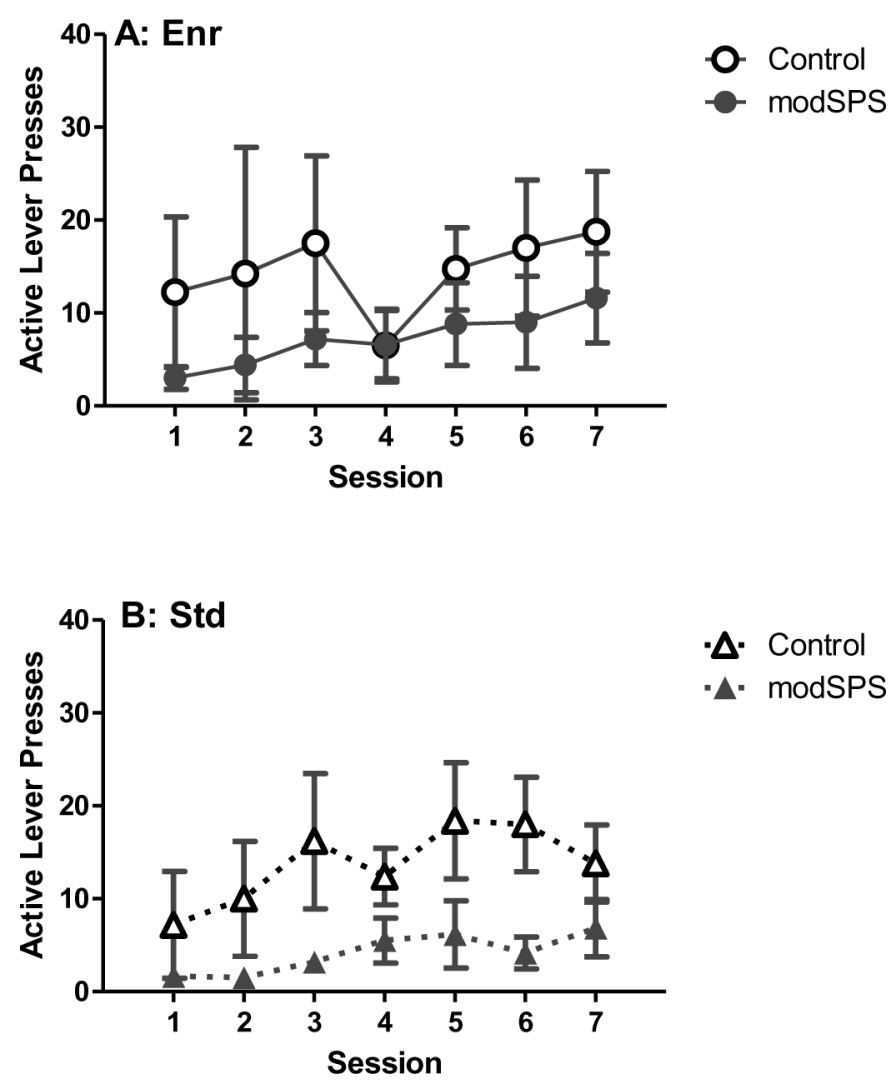

$\Delta \cdot$. Control

-A. modSPS

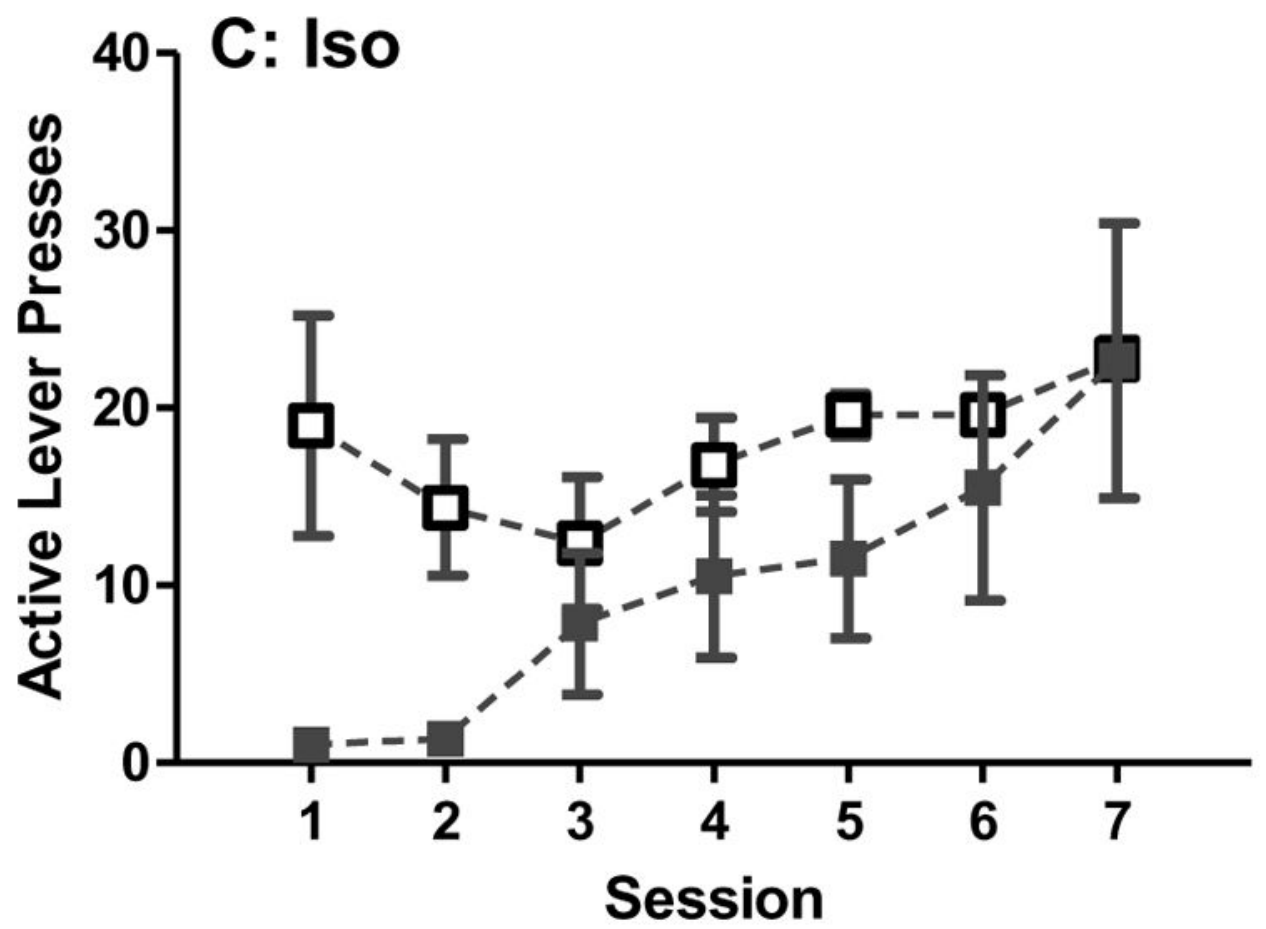

ㅁ. Control -1. modSPS

Figure 1. Acquisition of cocaine self-administration after modSPS

Behav Brain Res. Author manuscript; available in PMC 2019 February 15. 
Mean active lever presses ( \pm SEM) across sessions during acquisition of cocaine selfadministration ( $0.56 \mathrm{mg} / \mathrm{kg} /$ infusion) in (A) Enr rats, (B) Std rats, and (C) Iso rats exposed to modSPS (black symbols) or control (white symbols). 

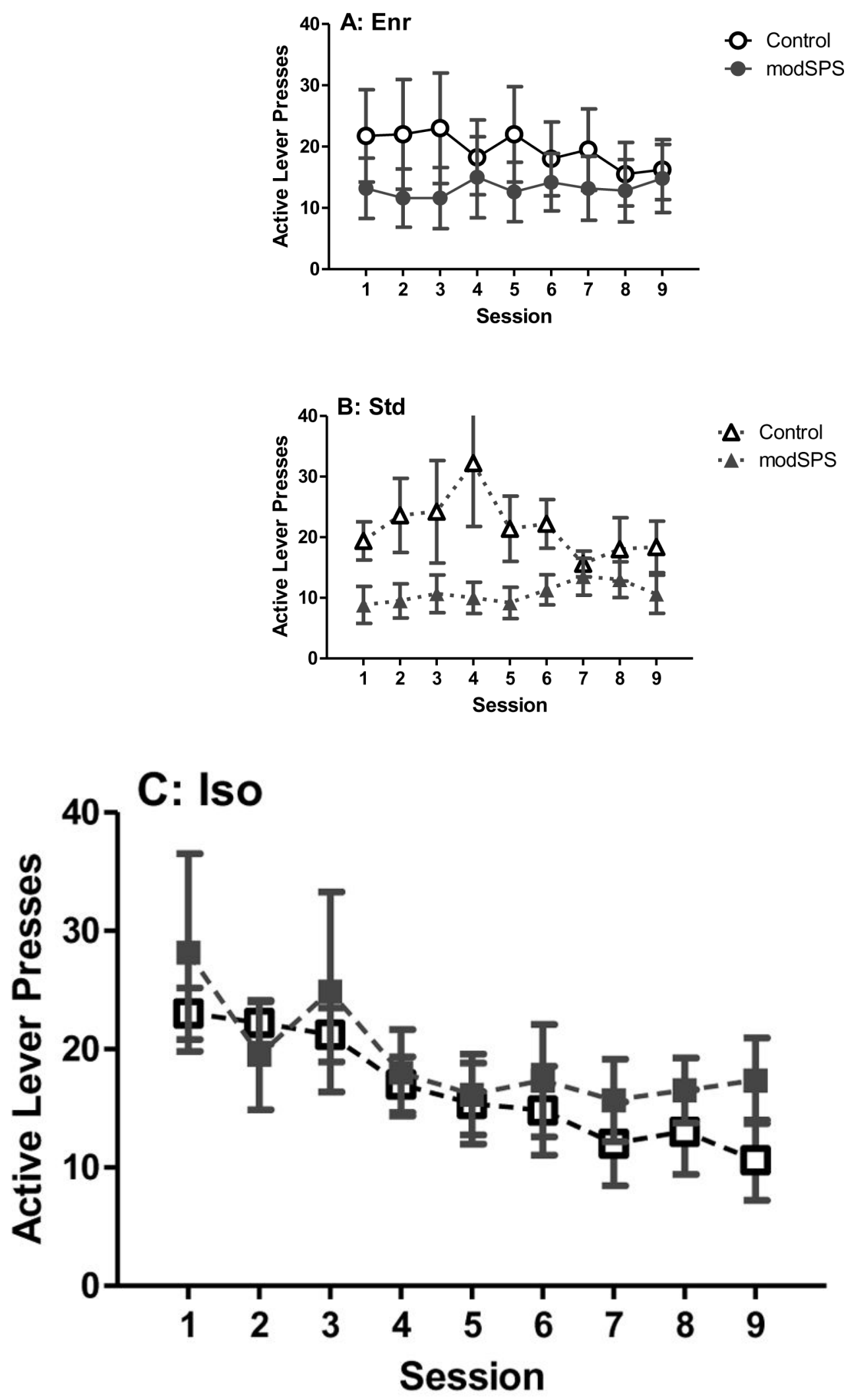

ㅁ. Control ㅁ. modSPS

Behav Brain Res. Author manuscript; available in PMC 2019 February 15. 
Figure 2. Maintenance of cocaine self-administration after modSPS

Mean active lever presses ( \pm SEM) across sessions during maintenance of cocaine selfadministration (0.56 mg/kg/infusion) in (A) Enr rats, (B) Std rats, and (C) Iso rats exposed to modSPS (black symbols) or control (white symbols). 

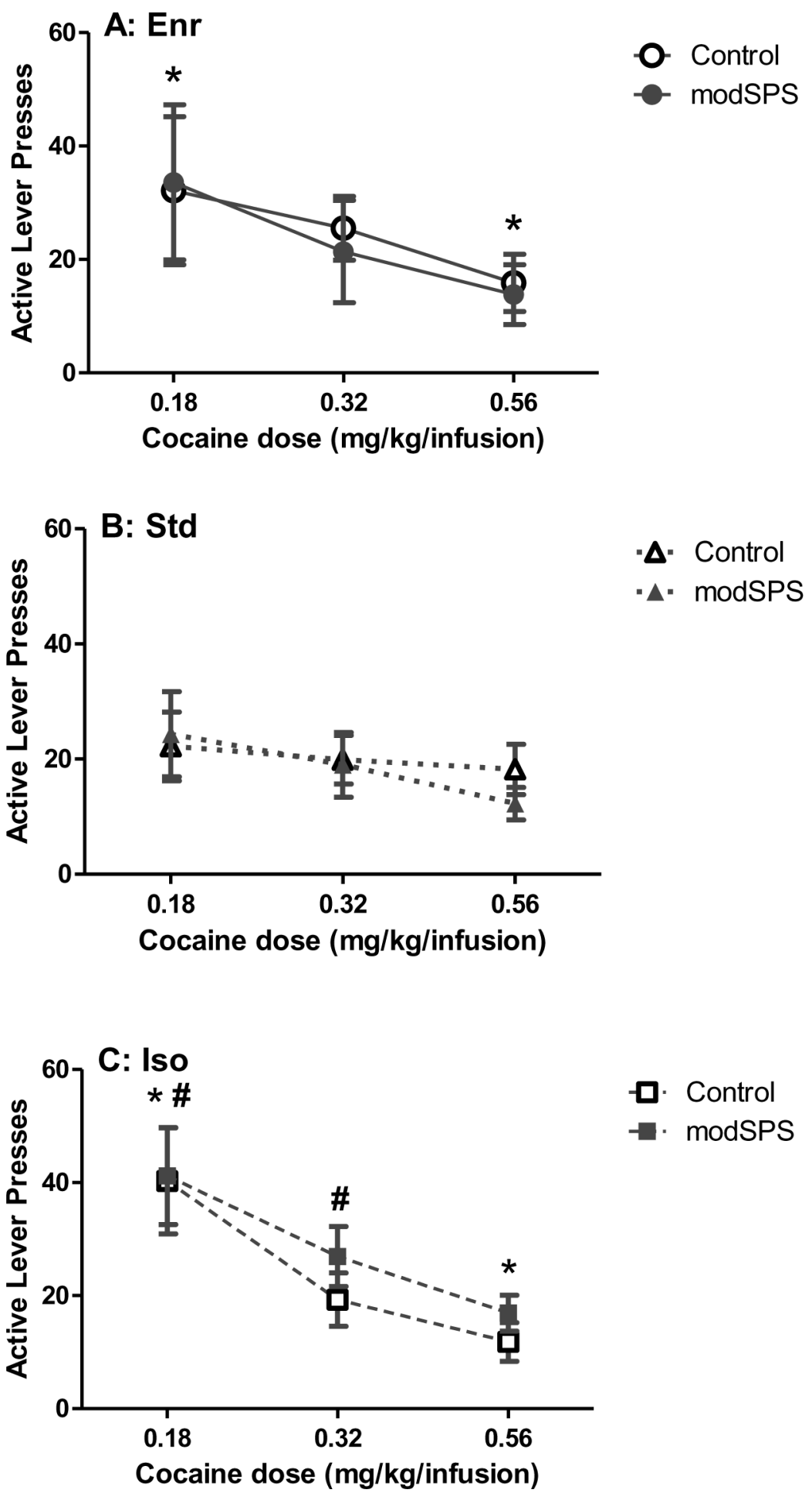

Figure 3. Cocaine dose-response curve after modSPS

Mean active lever presses ( \pm SEM) at $0.18 \mathrm{mg} / \mathrm{kg} /$ infusion, $0.32 \mathrm{mg} / \mathrm{kg} /$ infusion, and 0.56 $\mathrm{mg} / \mathrm{kg} /$ infusion cocaine in (A) Enr rats, (B) Std rats, and (C) Iso rats exposed to modSPS 
(black symbols) or control (white symbols). Matching symbols denote significant difference between doses, all $p<0.05$. 

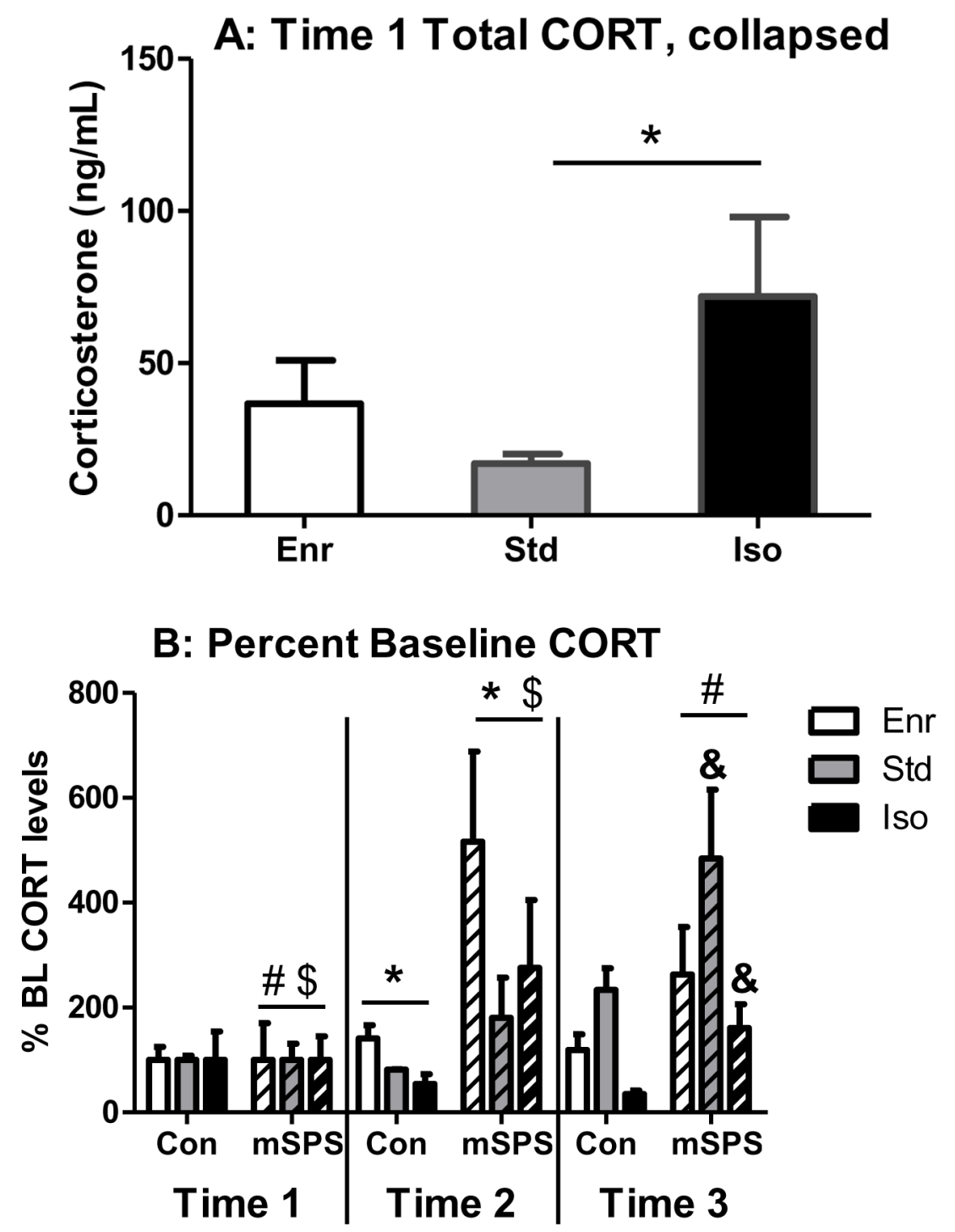

Figure 4. CORT release after modSPS

(A) Basal CORT levels in Enr, Std, and Iso rats at Time 1 (1 hr before modSPS). Data was collapsed across modSPS and control rats. (*) represents significant difference between Std and Iso, $p<0.05$. (B) Percent basal CORT in Enr, Std, and Iso rats at Time 1 (1 hr before modSPS), Time 2 (immediately following modSPS), and Time 3 (1 hr after modSPS). Enr (white bars), Std (gray bars), Iso (black bars), modSPS (mSPS; diagonally striped bars), control (Con; solid bars). Matching symbols denote significant difference between groups, all $p<0.05$. 

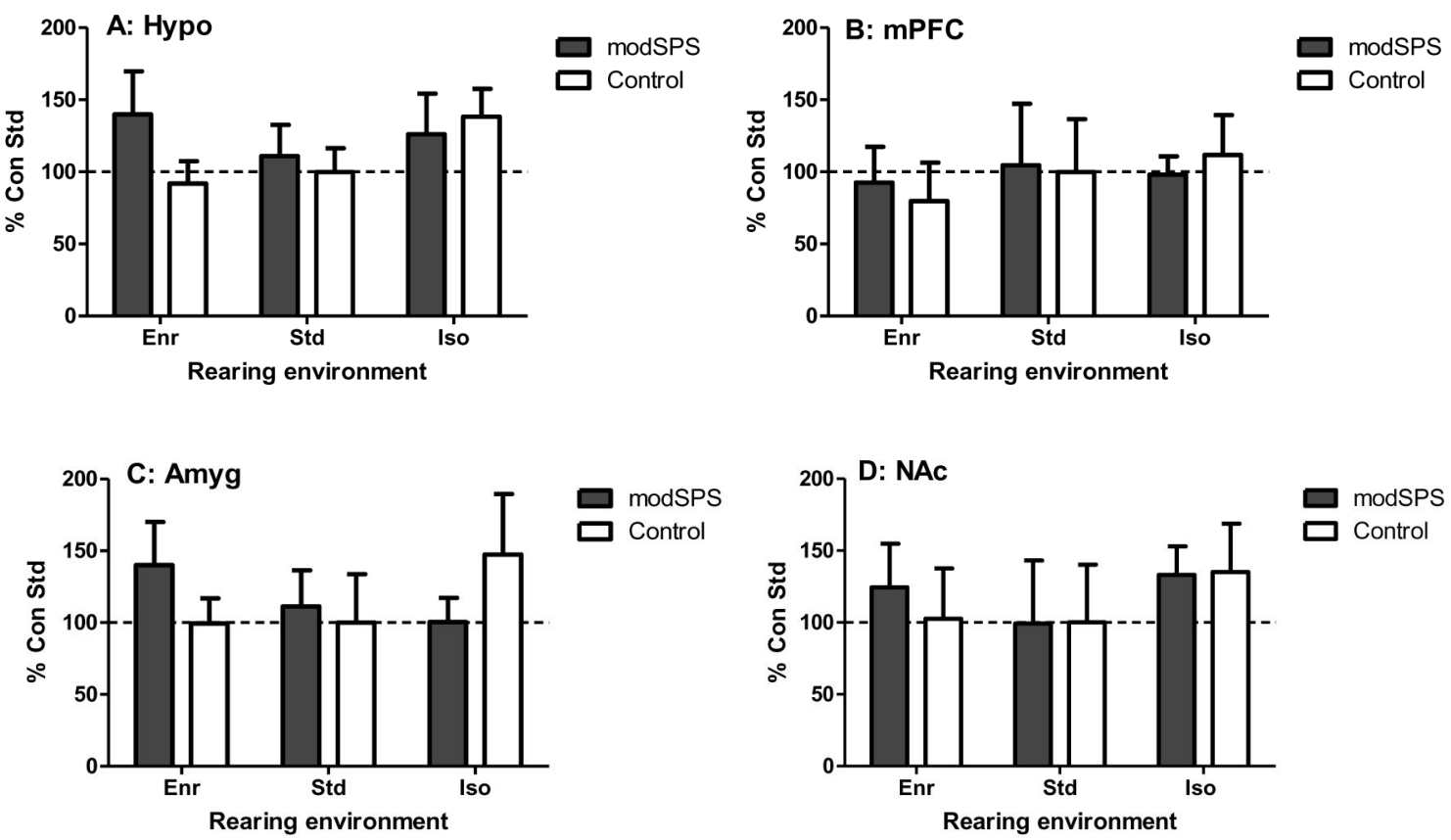

\section{E. Example Hypo Blot}

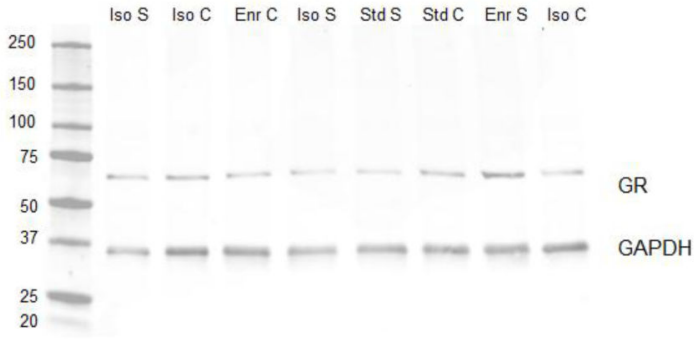

G. Example Amyg Blot

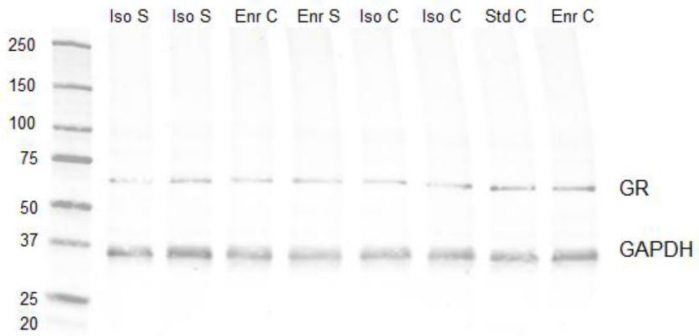

\section{F. Example mPFC Blot}

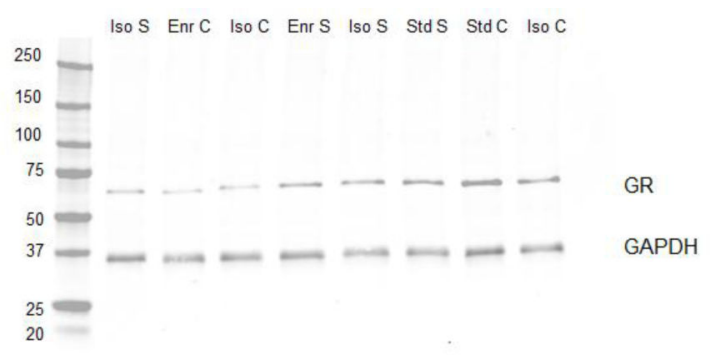

H. Example NAc Blot

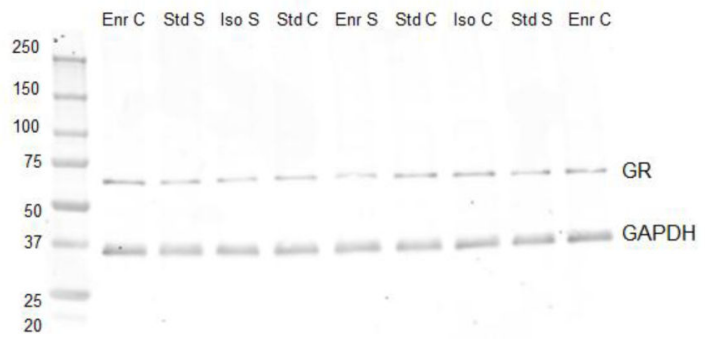

Figure 5. GR expression after modSPS

Percent control (control Std) GR expression in Enr, Std, and Iso rats exposed to modSPS or control in (A) hypothalamus; Hypo, (B) medial prefrontal cortex; mPFC, (C) amygdala; Amyg, and (D) nucleus accumbens; NAc. (E) Representative blot from Hypo. (F) Representative blot from mPFC, (G) Representative blot from Amyg, (H) Representative blot from NAc. Black bars: modSPS, White bars: control. 